\title{
Laplace Transform, Non-Constant Coefficients Differential Equations and Applications to Riccati Equation
}

\author{
Malick Ndiaye \\ Mathematics and Computer Sciences Department at Marist College, Poughkeepsie, NY, USA \\ Email: malick.ndiaye@marist.edu
}

How to cite this paper: Ndiaye, M. (2020) Laplace Transform, Non-Constant Coefficients Differential Equations and Applications to Riccati Equation. Applied Mathematics, 11, 639-649. https://doi.org/10.4236/am.2020.117043

Received: February 10, 2020

Accepted: July 19, 2020

Published: July 22, 2020

Copyright ( 2020 by author(s) and Scientific Research Publishing Inc. This work is licensed under the Creative Commons Attribution International License (CC BY 4.0).

http://creativecommons.org/licenses/by/4.0/

\begin{abstract}
In this paper, the Laplace Transform is used to find explicit solutions of a family of second order Differential Equations with non-constant coefficients. For some of these equations, it is possible to find the solutions using standard techniques of solving Ordinary Differential Equations. For others, it seems to be very difficult indeed impossible to find explicit solutions using traditional methods. The Laplace transform could be an alternative way. An application on solving a Riccati Equation is given. Recall that the Riccati Equation is a non-linear differential equation that arises in many topics of Quantum Mechanics and Physics.
\end{abstract}

\section{Keywords}

Ordinary Differential Equations, Laplace Transform

\section{Introduction}

The concept of Laplace Transform has been intensively used in diverse areas of Science and Engineering, for instance in electric circuit analysis, in communication engineering [1] [2] [3] [4].

It is also a powerful mathematical tool to solve non-homogeneous constant coefficients linear differential equations, especially when the forcing represents a discontinuous function as the Heaviside function or Dirac function [5] [6].

Like many operators, the Laplace Transform has the ability to change any ordinary linear differential equations with constant coefficients into algebraic equations.

It has been already used to find the explicit solutions of some non-constant coefficients linear differential equations. In [7], the authors used a new version 
of the Laplace Transform called the Sumudu Transform to find the explicit solution of the following non-constant coefficients differential equations along with their initial conditions:

$$
\begin{gathered}
y^{\prime \prime}+3 t y^{\prime}-6 y=2, \quad y(0)=0, \quad y^{\prime}(0)=0 \quad \text { solution } y=t^{2} \\
t y^{\prime \prime}-t y^{\prime}+y=2, \quad y(0)=2, \quad y^{\prime}(0)=-4 \quad \text { solution } y=2-4 t
\end{gathered}
$$

A similar work was performed in [8] and [9], where the authors used a different version of the Laplace Transform, this time called the Elkazi Transform, to find the explicit solutions of the initial value problems:

$$
\begin{array}{clll}
y^{\prime \prime}+t y^{\prime}-y=0, \quad y(0)=0, \quad y^{\prime}(0)=1, \quad t>0 & \text { solution } y=t \\
t y^{\prime \prime}+(1-2 t) y^{\prime}-2 y=0, \quad y(0)=1, \quad y^{\prime}(0)=2 & \text { solution } y=\mathrm{e}^{2 t} \\
t^{2} y^{\prime \prime}+4 t y^{\prime}+2 y=12 t^{2}, \quad y(0)=0, \quad y^{\prime}(0)=0 & \text { solution } y=t^{2}
\end{array}
$$

It would be very difficult, indeed impossible, to find explicit solutions to some of these types of initial value problems using standard methods. In this paper, the Laplace Transform is used to solve analytically a family of non-constant coefficients second order linear differential equations. In general, non-constant coefficients differential equations are still very difficult to be solved analytically. All the initial value problems listed above are particular cases of the family of non-constant linear differential equations found in this paper.

The paper is divided into five sections.

In Section 2, the concepts, properties and the existence of the Laplace transform are introduced.

In Section 3, conditions under which a family of non-constant coefficients ordinary differential equations can be solved quantitatively by using the Laplace Transform will be discussed. Specific examples of non-constant coefficients differential equations that satisfy those conditions will be given.

Section 4 gives an application to the Riccati Equation.

Section 5 is dedicated to the conclusion.

\section{Definition, Existence and Properties of Laplace Transform}

Definition 1. The Laplace Transform of a function $f$ denoted $\mathcal{L}(f)$ is given by the improper integral:

$$
\mathcal{L}(f)=\int_{0}^{\infty} f(t) \mathrm{e}^{-s t} \mathrm{~d} t=F(s) .
$$

for all numbers $s$ for which this improper integral converges.

The existence of the Laplace Transform of a given function has been discussed in [1] [2].

The following theorem gives a large class of functions for which the Laplace Transform exists, that is the improper integral converges.

Theorem 1. If $f$ is continuous on $(0, \infty)$ and $|f(t)| \leq M \mathrm{e}^{a t}$ then the Laplace Transform of $f, F(s)=\mathcal{L}(f)=\int_{0}^{\infty} f(t) \mathrm{e}^{-s t} \mathrm{~d} x$ exists for $s>a$.

Definition 2. The functions that fit the requirement of the hypothesis of the 
above theorem are said to be of exponential order $a$.

Next are examples of functions of exponential order.

Example 1. $f(t)=\mathrm{e}^{b t}, f(t)=\mathrm{e}^{b t} \sin (\omega t), \quad f(t)=\mathrm{e}^{b t} \cos (\omega t)$.

Remark 1. The function $f(t)=\mathrm{e}^{t^{2}}$ is not of exponential order since $\lim _{t \rightarrow \infty} \frac{\mathrm{e}^{t^{2}}}{M \mathrm{e}^{a t}}=\infty$ for all $M, a \in \mathbb{R}^{+}$.

Remark 2. As a practical matter, most of the functions encountered in the applications are of exponential order.

Theorem 2. If $f$ is of exponential order then $f^{(n)}$ is of exponential order for all $n$.

Proof. We just have to show that if $f$ is of exponential order then $f^{\prime}$ is of exponential order.

Suppose that $f^{\prime}$ is of exponential order. Then we have

$$
\begin{aligned}
\left|f^{\prime}(t)\right| & =\left|\lim _{h \rightarrow 0} \frac{f(t+h)-f(t)}{h}\right|=\lim _{h \rightarrow 0}\left|\frac{f(t+h)-f(t)}{h}\right| \\
& \leq \lim _{h \rightarrow 0} \frac{M \mathrm{e}^{a(t+h)}-M \mathrm{e}^{a t}}{h}=M \mathrm{e}^{a t} \lim _{h \rightarrow 0} \frac{\mathrm{e}^{a h}-1}{h} .
\end{aligned}
$$

Thus $\left|f^{\prime}(t)\right| \leq a M \mathrm{e}^{a t}$. By Induction, we can prove that $\left|f^{(n)}(t)\right| \leq a^{n} M \mathrm{e}^{a t}$, which shows that $f^{n}$ is of exponential order.

Next are some important properties of the Laplace Transform:

Property 1. The Laplace Transform is linear. That is,

$$
\mathcal{L}(f+g)=\mathcal{L}(f)+\mathcal{L}(g) \text { and } \mathcal{L}(\alpha f)=\alpha \mathcal{L}(f)
$$

Property 2. If $f$ is of exponential order then $\lim _{s \rightarrow \infty} F(s)=0$

Proof. $F(s)=\int_{0}^{\infty} \mathrm{e}^{-s t} f(t) \mathrm{d} t$. Since $f$ is of exponential order that is $|f(t)| \leq M \mathrm{e}^{a t}$ therefore $|F(s)| \leq \frac{1}{s-a}, s>a$. This shows that $\lim _{s \rightarrow \infty} F(s)=0$.

Property 3. If a continuous function $f$ on $[0, \infty]$ has Laplace Transform $F(s)$ then $f$ is the only function whose Laplace Transform is $F(s)$.

Proof. We need to show that if $\int_{0}^{\infty} \mathrm{e}^{-s t} f(t) \mathrm{d} t=\int_{0}^{\infty} \mathrm{e}^{-s t} g(t) \mathrm{d} t$ then $f(t)=g(t)$, $s>a, a>0$.

$$
\int_{0}^{\infty} \mathrm{e}^{-s t} f(t) \mathrm{d} t=\int_{0}^{\infty} \mathrm{e}^{-s t} g(t) \mathrm{d} t \text { then } \int_{0}^{\infty} \mathrm{e}^{-s t}(f(t)-g(t)) \mathrm{d} t=0 .
$$

Let's make the change $x=\mathrm{e}^{-t}, \mathrm{~d} t=-\frac{1}{x} \mathrm{~d} x$,

$$
\begin{gathered}
\int_{0}^{\infty} \mathrm{e}^{-s t}(f(t)-g(t)) \mathrm{d} t=\int_{0}^{1} x^{s}(f(-\ln x)-g(-\ln x)) \mathrm{d} x . \\
s>a, \quad s-a-1 \geq 0 . \\
\int_{0}^{1} x^{a+1}(f(-\ln x)-g(-\ln x)) x^{s-a-1} \mathrm{~d} x=\int_{0}^{1} x^{a+1} M(-\ln x) x^{s-a-1} \mathrm{~d} x=0,
\end{gathered}
$$

where

$$
M(t)=f(t)-g(t)
$$

Define 


$$
F(x)=x^{a+1} M(-\ln x) \text { if } x \in(0,1]
$$

and

$$
F(0)=0
$$

So

$$
\lim _{x \rightarrow 0^{+}} F(x)=\lim _{x \rightarrow 0^{+}} x^{a+1} M(-\ln x)=\lim _{t \rightarrow \infty} \mathrm{e}^{-(a+1) t} M(t)=0 .
$$

Since $M$ is of exponent order.

So $\lim _{x \rightarrow 0^{+}} F(x)=F(0)$ then $F$ is continuous on $[0,1]$. According to the Weierstrass Approximation theorem, any continuous function can be approximated as closely as desired by a polynomial function. That is there exists a sequence of polynomials $P_{n}(x)=a_{0}^{n}+a_{1}^{n} x+a_{2}^{n} x^{2}+a_{3}^{n} x^{3}+\cdots+a_{m}^{n} x^{m}$ such that $\lim _{n \rightarrow \infty} P_{n}(x)=F(x)$.

$$
\int_{0}^{1} F(x) P_{n}(x) \mathrm{d} x=\sum_{k=0}^{m} a_{k}^{n} \int_{0}^{1} F(x) x^{k}=0
$$

then

$$
\begin{gathered}
\lim _{n \rightarrow \infty} \int_{0}^{1} F(x) P_{n}(x) \mathrm{d} x=\int_{0}^{1} F(x) \lim _{n \rightarrow \infty} P_{n}(x) \mathrm{d} x=\int_{0}^{1} F(x)^{2} \mathrm{~d} x=0 \\
\Rightarrow F(x)=0
\end{gathered}
$$

We conclude that

$$
x^{a+1}(f(-\ln x)-g(-\ln x))=0 \Rightarrow f(-\ln x)-g(-\ln x)=0
$$

therefore $f(t)=g(t)$.

This property means that if $f$ is continuous on $[0, \infty)$ and if $\mathcal{L}(f)=F$ then $f=M(F)$.

$M$ is called the inverse of $\mathcal{L}$ and $M=\mathcal{L}^{-1}$.

$\mathcal{L}^{-1}$ is also linear.

\section{Explicit Solutions for a Family of Non-Constant Coefficients Linear Second Order Differential Equations}

The goal in this section is to solve analytically non-constant coefficients linear second order differential equations:

$$
q_{2}(t) y^{\prime \prime}+q_{1}(t) y^{\prime}+q_{0}(t) y=g(t),
$$

using the Laplace Transform where $q_{0}(t), q_{1}(t)$ and $q_{2}(t)$ are functions of t. Specifically, conditions on $q_{0}, q_{1}$ and $q_{2}$ will be stated so that the second order differential equations will have explicit solutions, that is expressed in term of elementary functions.

Proposition 1. If $q(t), y(t) \in C^{2}(a, b)$ then

$$
\begin{aligned}
\mathcal{L}\left(q(t) y^{\prime}\right)= & -\mathcal{L}\left(q^{\prime}(t) y\right)+s \mathcal{L}(q(t) y)-q(0) y(0) \\
\mathcal{L}\left(q(t) y^{\prime \prime}\right)= & \mathcal{L}\left(q^{\prime \prime}(t) y\right)-2 s \mathcal{L}\left(q^{\prime}(t) y\right)+s^{2} \mathcal{L}(q(t) y) \\
& -q(0) y^{\prime}(0)+q^{\prime}(0) y(0)-s q(0) y(0)
\end{aligned}
$$


Proof. $\mathcal{L}\left(q(t) y^{\prime}\right)=\int_{0}^{\infty} q(t) y^{\prime} \mathrm{e}^{-s t} \mathrm{~d} t=\lim _{b \rightarrow \infty} \int_{0}^{b} q(t) y^{\prime} \mathrm{e}^{-s t} \mathrm{~d} t$.

Using integration by part and the fact that $y$ is of exponential order, we obtain

$$
\mathcal{L}\left(q(t) y^{\prime}\right)=-\mathcal{L}\left(q^{\prime}(t) y\right)+s \mathcal{L}(q(t) y)-q(0) y(0) .
$$

We can use (1), the fact that $y^{\prime}$ is of exponential order and Theorem 2 to find (2).

First, we have the following

$$
\mathcal{L}\left(q(t) y^{\prime \prime}\right)=-\mathcal{L}\left(q^{\prime}(t) y^{\prime}\right)+s \mathcal{L}\left(q(t) y^{\prime}\right)-q(0) y^{\prime}(0)
$$

Using (1) on $\mathcal{L}\left(q^{\prime}(t) y^{\prime}\right)$ and on $\mathcal{L}\left(q(t) y^{\prime}\right)$, we obtain (2). That is

$$
\begin{aligned}
\mathcal{L}\left(q(t) y^{\prime \prime}\right)= & \mathcal{L}\left(q^{\prime \prime}(t) y\right)-2 s \mathcal{L}\left(q^{\prime}(t) y\right)+s^{2} \mathcal{L}(q(t) y) \\
& -y^{\prime}(0) q(0)+y(0) q^{\prime}(0)-s q(0) y(0)
\end{aligned}
$$

Theorem 3. Let $(E): q_{2}(t) y^{\prime \prime}+q_{1}(t) y^{\prime}+q_{0}(t) y=g(t)$ be a non-constant coefficients non-homogeneous linear differential equation with $q_{0}(t), q_{1}(t)$, $q_{2}(t)$ and $g(t) \in C^{2}(a, b)$.

Under the following conditions.

$$
\begin{aligned}
q_{0}(t)= & \left(c_{1} t+c_{2}\right) q_{2}(t)+\left(c_{3} t+c_{4}\right) q_{2}^{\prime}(t)+q_{2}^{\prime \prime}(t)+c_{5}, \\
& q_{1}(t)=\left(c_{3} t+c_{4}\right) q_{2}(t)+c_{6} q_{2}^{\prime}(t)+c_{7} \\
& c_{5}=0, c_{7}=0, c_{6}=2, c_{1} \neq 0 \text { or } c_{3} \neq 0
\end{aligned}
$$

and $q_{2}(t)$ is neither a constant nor a linear function, where $c_{1}, c_{2}, c_{3}, c_{4}, c_{5}, c_{6}$ and $c_{7}$ are constant real numbers, the Laplace transform of $q_{2}(t) y$ is given by

$$
\mathcal{L}\left(q_{2}(t) y\right)=\mathrm{e}^{h(s)} \int\left(-\mathrm{e}^{-h(s)}\right) \frac{f(s)}{c_{3} s+c_{1}} \mathrm{~d} s+c \mathrm{e}^{h(s)}
$$

where

$$
\begin{gathered}
f(s)=\mathcal{L}(g(t))+q_{2}(0) y^{\prime}(0)-q_{2}^{\prime}(0) y(0)+s q_{2}(0) y(0)+q_{1}(0) y(0) \\
h(s)=\int \frac{s^{2}+c_{4} s+c_{2}-c_{3}}{c_{3} s+c_{1}} \mathrm{~d} s
\end{gathered}
$$

and $c$ is a constant.

Therefore the explicit solution of $(E)$ is given by:

$$
y(t)=\frac{1}{q_{2}(t)} \mathcal{L}^{-1}\left(\mathrm{e}^{h(s)} \int\left(-\mathrm{e}^{-h(s)}\right) \frac{f(s)}{c_{3} s+c_{1}} \mathrm{~d} s+c \mathrm{e}^{h(s)}\right)
$$

Proof. The first step is to take the Laplace Transform of both sides of (E).

Using the linearity of the Laplace Transform, we get:

$$
\mathcal{L}\left(q_{2}(t) y^{\prime \prime}\right)+\mathcal{L}\left(q_{1}(t) y^{\prime}\right)+\mathcal{L}\left(q_{0}(t) y\right)=\mathcal{L}(g(t))
$$

Now using proposition 1 , we have

$$
\begin{aligned}
& \mathcal{L}\left(q_{2}^{\prime \prime}(t) y\right)-2 s \mathcal{L}\left(q_{2}^{\prime}(t) y\right)+s^{2} \mathcal{L}\left(q_{2}(t) y\right)-\mathcal{L}\left(q_{1}^{\prime}(t) y\right)+s \mathcal{L}\left(q_{1}(t) y\right) \\
& +\mathcal{L}\left(q_{0}(t) y\right)-q_{2}(0) y^{\prime}(0)+q_{2}^{\prime}(0) y(0)-s q_{2}(0) y(0)-q_{1}(0) y(0) \\
& =\mathcal{L}(g(t))
\end{aligned}
$$


Using (3), (4) and (5) in (8), and after simplification, we obtain the following equation:

$$
\begin{aligned}
& \left(c_{3} s+c_{1}\right) \mathcal{L}\left(t q_{2}(t) y\right)+\left(s^{2}+c_{4} s+c_{2}-c_{3}\right) \mathcal{L}\left(q_{2}(t) y\right) \\
& -y^{\prime}(0) q_{2}(0)+y(0) q_{2}^{\prime}(0)=\mathcal{L}(g(t))
\end{aligned}
$$

Since $\mathcal{L}\left(t q_{2}(t) y\right)=-\frac{\mathrm{d}}{\mathrm{d} s} \mathcal{L}\left(q_{2}(t) y\right)$ therefore (9) implies the following first order differential equation:

$$
-\left(c_{3} s+c_{1}\right) \frac{\mathrm{d}}{\mathrm{ds}} \mathcal{L}\left(q_{2}(t) y\right)+\left(s^{2}+c_{4} s+c_{2}-c_{3}\right) \mathcal{L}\left(q_{2}(t) y\right)=f(s)
$$

where

$$
f(s)=\mathcal{L}(g(t))+y^{\prime}(0) q_{2}(0)-y(0) q_{2}^{\prime}(0)+s q_{2}(0) y(0)+q_{1}(0) y(0)
$$

Using the integrating factor method, we can find $\mathcal{L}\left(q_{2}(t) y\right)$ and therefore the solution of (E) under the conditions stated in the theorem.

Let's turn now our attention to simple examples for which the conditions of the theorem 3 are satisfied.

Example 2. We suppose the $c_{1}=0, c_{1}=0, c_{3}=1, c_{4}=-2, y(0)=0$, $y^{\prime}(0)=0$ and $g(t)=-1+2 t$ so

$$
\begin{gathered}
q_{0}(t)=-2 q_{2}(t)+(t-2) q_{2}^{\prime}(t)+q_{2}^{\prime \prime}(t) . \\
q_{1}(t)=(t-2) q_{2}(t)+2 q_{2}^{\prime}(t) .
\end{gathered}
$$

We deal with the family of non-constant coefficients linear ordinary differential equations.

$$
\begin{aligned}
& q_{2}(t) y^{\prime \prime}+\left((t-2) q_{2}(t)+2 q_{2}^{\prime}(t)\right) y^{\prime} \\
& +\left(-2 q_{2}(t)+(t-2) q_{2}^{\prime}(t)+q_{2}^{\prime \prime}(t)\right) y=-1+2 t
\end{aligned}
$$

We can see that the explicit solution of this equation is given by:

$$
y(t)=\frac{t^{2}}{2 q_{2}(t)} .
$$

Using (6) we obtain:

$$
\mathcal{L}\left(q_{2}(t) y\right)=\frac{1}{s^{3} \mathrm{e}^{-\frac{s^{2}}{2}+2 s}} \int(-s+2) \mathrm{e}^{-\frac{s^{2}}{2}+2 s} \mathrm{~d} s+\frac{c}{s^{3} \mathrm{e}^{-\frac{s^{2}}{2}+2 s}}
$$

where $c$ is given by the initial conditions. Therefore $\mathcal{L}\left(q_{2}(t) y\right)=\frac{1}{s^{3}}+c \frac{\mathrm{e}^{\frac{s^{2}}{2}-2 s}}{s^{3}}$. So using the linearity of $\mathcal{L}^{-1}$

$$
y=\frac{1}{q_{2}(t)} \mathcal{L}^{-1}\left(\frac{1}{s^{3}}\right)+c \mathcal{L}^{-1}\left(\frac{\mathrm{e}^{\frac{s^{2}}{2}-2 s}}{s^{3}}\right)
$$

$\mathcal{L}^{-1}\left(\frac{1}{s^{3}}\right)=\frac{t^{2}}{2}$ but $\mathcal{L}^{-1}\left(\frac{\mathrm{e}^{\frac{s^{2}}{2}-2 s}}{s^{3}}\right)$ seems to be very complicated to be found, 
however we can use the following argument to find $c$. We know that $\lim _{s \rightarrow \infty} \frac{1}{s^{3}}=0, \quad \lim _{s \rightarrow \infty} \frac{\mathrm{e}^{\frac{s^{2}}{2}-2 s}}{s^{3}}=\infty$ and according to property 2, $\lim _{s \rightarrow \infty} \mathcal{L}\left(q_{2}(t) y\right)=0$ therefore $c=0$ and $y(t)=\frac{t^{2}}{2 q_{2}(t)}$.

Example 3. Choosing $c_{1}=1, \quad c_{2}=-2, \quad c_{3}=1, \quad c_{4}=2, y(0)=0$, $y^{\prime}(0)=0$ and then

$$
\begin{gathered}
q_{0}(t)=(t-2) q_{2}(t)+(t+2) q^{\prime}(t)+q_{2}^{\prime \prime}(t) . \\
q_{1}(t)=(t+2) q_{2}(t)+2 q_{2}^{\prime}(t) .
\end{gathered}
$$

and we get the following equation:

$$
\begin{aligned}
& q_{2}(t) y^{\prime \prime}+\left((t+2) q_{2}(t)+2 q_{2}^{\prime}(t)\right) y^{\prime} \\
& +\left((t-2) q_{2}(t)+(t+2) q_{2}^{\prime}(t)+q_{2}^{\prime \prime}(t)\right) y=t \mathrm{e}^{-t}
\end{aligned}
$$

Using the same procedure as in example 2, The explicit solution can be written as:

$$
y(t)=-\frac{t^{3} \mathrm{e}^{-t}}{3 ! q_{2}(t)}
$$

Theorem 4. Under the conditions (3), (4), $c_{5}=0, c_{7}=0, c_{6}=2, c_{1}=0$, $c_{3}=0$ and $q_{2}(t)$ is not linear function, the Laplace Transform of $q_{2}(t) y$ is given by:

$$
\mathcal{L}\left(q_{2}(t) y\right)=\frac{f(s)}{s^{2}+c_{4} s+c_{2}}
$$

where $f(s)$ is given by (7) therefore

$$
y(t)=\frac{1}{q_{2}(t)} \mathcal{L}^{-1}\left(\frac{f(s)}{s^{2}+c_{4} s+c_{2}}\right)
$$

and under the conditions (3), (4), $c_{1}=0, c_{3}=0, q_{2}(t)=c_{9} t+c_{10}$

$$
\mathcal{L}(y)=\mathrm{e}^{v(s)} \int\left(-\mathrm{e}^{-v(s)}\right) \frac{f(s)}{c_{9} s^{2}+c_{4} c_{9} s+c_{2} C_{9}} \mathrm{~d} s+k \mathrm{e}^{v(s)}
$$

where

$$
v(s)=\int \frac{c_{10} s^{2}+\left(-2 c_{9}+c_{4} c_{10}+c_{6} c_{9}+c_{7}\right) s+{ }_{2} c_{10}+c_{5}}{c_{9} s^{2}+c_{4} c_{9} s+c_{2} c_{9}} \mathrm{~d} s
$$

and where $f(s)$ is given by $(7)$.

Therefore the solution to $(E)$ is given by:

$$
y(t)=\mathcal{L}^{-1}\left(\mathrm{e}^{v(s)} \int\left(-\mathrm{e}^{-v(s)}\right) \frac{f(s)}{c_{9} s^{2}+C_{4} C_{9} s+C_{2} c_{9}} \mathrm{~d} s+k \mathrm{e}^{v(s)}\right)
$$

Proof. The proof is straightforward and is done the same way as in theorem 3.

We can also notice that while the conditions (3), (4), $c_{1}=0, c_{3}=0$, 
$q_{2}(t)=c_{9} t+c_{10}$ lead to a first order differential equation, the conditions (3), (4), $c_{5}=0, c_{7}=0, c_{6}=2, c_{1}=0$, and $q_{2}(t)$ is not linear function simply lead to an algebraic equation.

Example 4. Under the conditions (3), (4), $c_{5}=0, c_{7}=0, c_{6}=2, c_{1}=0$, $c_{3}=0, c_{2}=-3, c_{4}=2$ and $q_{2}(t)$ is not a linear function, we get the following non-constant linear differential equation:

$$
q_{2}(t) y^{\prime \prime}+\left(c_{4} q_{2}(t)+2 q_{2}^{\prime}(t)\right) y^{\prime}+\left(c_{2} q_{2}(t)+c_{4} q_{2}^{\prime}(t)+q_{2}^{\prime \prime}(t)\right) y=g(t)
$$

Letting $g(t)=2$. Using $(10)$, we find

$$
y=\frac{1}{q_{2}(t)} \mathcal{L}^{-1}\left(-\frac{2}{3} \frac{1}{s}+\frac{1}{2} \frac{1}{(s-1)}+\frac{1}{6} \frac{1}{(s+3)}\right)
$$

then

$$
y=\frac{1}{q_{2}(t)}\left(-\frac{2}{3}+\frac{1}{2} \mathrm{e}^{t}+\frac{1}{6} \mathrm{e}^{-3 t}\right)
$$

Notice that this solution could be easily found by changing Equation (12) into this following linear equation:

$$
\frac{\mathrm{d}^{2}}{\mathrm{~d} t^{2}}\left(q_{2}(t) y\right)+c_{4} \frac{\mathrm{d}}{\mathrm{d} t}\left(q_{2}(t) y\right)+c_{2}\left(q_{2}(t) y\right)=g(t)
$$

So using a traditional method, we can find exactly the same result.

Now focusing on the example listed in the introduction, we can show that they all can be obtained using the conditions described in the theorem 4 or theorem 5 given later.

Example 5. Under the conditions (3), (4), $c_{5}=0, c_{7}=0, c_{6}=2, c_{1}=0$, $c_{3}=0, c_{2}=0, c_{4}=0, g(t)=12 t^{2}, y(0)=0, y^{\prime}(0)=0$, we obtained one of the initial problem listed in the introduction:

$$
t^{2} y^{\prime \prime}+4 t y^{\prime}+2 y=12 t^{2}, \quad y(0)=0, \quad y^{\prime}(0)=0
$$

Using (10), we obtain the same solution $y=t^{2}$.

Example 6. Under the condition (3), (4), $c_{1}=0, c_{3}=0, q_{2}(t)=c_{9} t+c_{10}$ and the additional ones: $c_{2}=0, c_{4}=-1, c_{9}=1, c_{10}=0, c_{5}=2, c_{6}+c_{7}=0$, $g(t)=2, y(0)=2, y^{\prime}(0)=-4$, we get one of the equation listed in the introduction: $t y^{\prime \prime}-t y^{\prime}+y=2$.

We obtain $y=2-4 t$ by using (11).

Same thing for the initial value problem listed in the introduction:

$$
t y^{\prime \prime}+(1-2 t) y^{\prime}-2 y=0, y(0)=1, \quad y^{\prime}(0)=2,
$$

we use the same above conditions (3), (4), $c_{1}=0, c_{3}=0, q_{2}(t)=c_{9} t+c_{10}$ and the additional conditions: $c_{2}=0, c_{4}=-2, c_{9}=1, c_{10}=0, c_{5}=0$, $c_{6}+c_{7}=1, g(t)=0$, Using (11), we find $y(t)=\mathrm{e}^{2 t}$.

Theorem 5. Under the conditions (3), (4), $c_{1} \neq 0$ or $c_{3} \neq 0$ and $q_{2}(t)$ is a constant function, that is $q_{2}(t)=c_{8} \neq 0$ then

$$
\mathcal{L}(y)=\mathrm{e}^{w(s)} \int\left(-\mathrm{e}^{-w(s)}\right) \frac{f(s)}{C_{3} c_{8} s+c_{1} c_{8}} \mathrm{~d} s+k \mathrm{e}^{w(s)}
$$


where $f(s)$ is given by $(7)$.

$$
w(s)=\int \frac{c_{8} s^{2}+\left(c_{4} c_{8}+c_{7}\right) s-c_{3} c_{8}+c_{2} c_{8}+c_{5}}{c_{3} c_{8} s+c_{1} c_{8}} \mathrm{~d} s
$$

and $k$ a constant.

Therefore

$$
y(t)=\mathcal{L}^{-1}\left(\mathrm{e}^{w(s)} \int\left(-\mathrm{e}^{-w(s)}\right) \frac{f(s)}{C_{3} C_{8} s+C_{1} C_{8}} \mathrm{~d} s+k \mathrm{e}^{w(s)}\right)
$$

Now under (3), (4), $c_{1}=0, c_{3}=0$ and $q_{2}(t)$ is a constant function, that is $q_{2}(t)=c_{8} \neq 0$ then

$$
\mathcal{L}(y)=\frac{f(s)}{c_{8} s^{2}+\left(c_{4} c_{8}+c_{7}\right) s+c_{2} c_{8}+c_{5}}
$$

therefore

$$
y=\mathcal{L}^{-1}\left(\frac{f(s)}{c_{8} s^{2}+\left(c_{4} c_{8}+c_{7}\right) s+c_{2} c_{8}+c_{5}}\right)
$$

Proof. The proof is straightforward and is done the same way as in theorem 3.

Example 7. Under the conditions (3), (4), $c_{1} \neq 0$ or $c_{3} \neq 0$ and $q_{2}(t)$ is a constant function, that is $q_{2}(t)=c_{8} \neq 0$ and additional conditions $c_{1}=0$, $c_{3}=3, \quad c_{2}+c_{5}=-6, \quad c_{4}+c_{7}=-6, g(t)=2, y(0)=0, y^{\prime}(0)=0$ we get one of the initial value problems listed in the introduction:

$$
y^{\prime \prime}+3 t y^{\prime}-6 y=2, \quad y(0)=0, \quad y^{\prime}(0)=0
$$

Using (14), we have the solution: $y=t^{2}$.

Example 8. Under the conditions (3), (4), $c_{1} \neq 0$ or $c_{3} \neq 0$ and $q_{2}(t)$ is a constant function, that is $q_{2}(t)=c_{8} \neq 0$ and additional conditions $c_{1}=0$, $c_{3}=1, \quad c_{2}+c_{5}=-1, \quad c_{4}=0, \quad c_{7}=0, g(t)=0, y(0)=0, y^{\prime}(0)=1$ we obtain one of the initial value problems listed in the introduction:

$$
y^{\prime \prime}+t y^{\prime}-y=0, \quad y(0)=0, \quad y^{\prime}(0)=1
$$

Using (14), we get the solution: $y=t$.

\section{Application to the Riccati Equation}

The Riccati Equation named after the mathematician Jacopo Francesco Riccati [10] is the simplest non-linear differential equation. It can be written as:

$$
\frac{\mathrm{d} y}{\mathrm{~d} t}=A(t) y^{2}+B(t) y+C(t)
$$

where $A(t), B(t)$ and $C(t)$ are functions of the independent variable $t$. The Riccati Equation naturally arises in many fields. Many equations in Physics, Cosmology and Quantum mechanics involve or can be changed into a Riccati Equation [11]. It is well known that there is no general way to solve the Riccati equation. When a particular solution $y_{1}$ is found for the Riccati equation, we can use the change $u=y-y_{1}$ to find the general solution of the Riccati Equa- 
tion. This change of variable turns the Riccati into a Bernouilli Equation with $u$ the unknown variable:

$$
\frac{\mathrm{d} u}{\mathrm{~d} t}+\left(-2 A(t) y_{1}-B(t)\right) u=A(t) u^{2}
$$

There are several ways to find a particular solution of a Riccati equation utilizing relations between the coefficients $A(t), B(t)$ and $C(t)$. In general, a change of variable is performed that turned the Riccati equation to a separable equation. There are several methods that can be found in [12].

It is also well-known that the Riccati Equation can be reduced to the non-constant coefficients homogeneous second order differential equation:

$$
A(t) \frac{\mathrm{d}^{2} u}{\mathrm{~d} x^{2}}+\left(-A(t) B(t)-\frac{\mathrm{d} A}{\mathrm{~d} t}\right) \frac{\mathrm{d} u}{\mathrm{~d} t}+A(t) C(t) u=0
$$

by using the substitution: $y=-\frac{u^{\prime}}{A(t) u}$.

The purpose of this application is to show an example as to how to solve the Riccati Equation using the Laplace Transform.

Example: Solving the Riccati Equation:

$$
\frac{\mathrm{d} y}{\mathrm{~d} t}=t y^{2}+\left(-\frac{2}{t}+2\right) y-\frac{2}{t^{2}}
$$

In this case: $A(t)=t, B(t)=-\frac{2}{t}+2, C(t)=-\frac{2}{t^{2}}$. Using the substitution $y=\frac{u^{\prime}}{A(t) u}$ where $A(t)=t$, we obtain the non-constant coefficients nonhomogeneous second order differential equation:

$$
t \frac{\mathrm{d}^{2} u}{\mathrm{~d} t^{2}}+(1-2 t) \frac{\mathrm{d} u}{\mathrm{~d} t}-2 u=0
$$

which correspond to Equation (13). A solution of this equation is given by $u(t)=\mathrm{e}^{2 t}$ therefore a solution of the Riccati equation is given by: $y_{1}=\frac{u^{\prime}}{t u}=-\frac{2}{t}$. Using the substitution: $w=y+\frac{2}{t}$, we find the general solution of the Riccati equation:

$$
y(t)=-t^{2} \mathrm{e}^{2 t} \int \frac{1}{t \mathrm{e}^{2 t}} d t+c t^{2} \mathrm{e}^{2 t}-\frac{2}{t}
$$

where $c$ is a constant.

\section{Conclusion}

In this paper, explicit solutions of non-constant coefficients ordinary differential equations are given by using the Laplace Transform. In fact, the main challenge at this point is to compute the inverse of the Laplace Transform of a given function. For most functions, the inverse of the Laplace transform is not listed in the table of Laplace Transform and it seems to be very difficult to evaluate. I was 
very fortunate to find cases in which the inverse of the Laplace Transform can be found easily and therefore, an explicit solution follows. Now for further research, we can try to figure out what change of variable would turn the Riccati Equation into a non-homogeneous non-constant coefficients second order differential equation. This would lead to more applications in solving the Riccati equation using the results from Section 2.

\section{Conflicts of Interest}

The author declares no conflicts of interest regarding the publication of this paper.

\section{References}

[1] Dyke, P. (2014) An Introduction to Laplace Transform and Fourier Series. Springer-Verlag, London. https://doi.org/10.1007/978-1-4471-6395-4

[2] Schiff, J.L. (1999) The Laplace Transform Theory and Applications. Springer-Verlag, New York. https://doi.org/10.1007/978-0-387-22757-3

[3] Stroud, K.A. (2003) Advanced Engineering Mathematics. cds.cern.chc.

[4] Dass, H.K. (2009) Advanced Engineering Mathematics. S.Chand and Company Ltd., New Delhi.

[5] Blanchard, P., Devaney, R.L. and Hall, G.R. (2012) Differential Equations. 4th Edition. Brooks/Cole, Boston.

[6] Misra, M. Laplace Transform. Hardwari Publications, Alla Habad (India).

[7] Hassan Eltayeb, A.K. and Kilicman, A. (2010) A Note on the Sunudu Transform and Differential Equations. Applied Mathematical Sciences , 4, 167-173.

[8] Elkazi, T.M. and Elkazi, S.M. (2011) On the Elkazi Transform and Ordinary Differential Equations with Variable Coefficients. Advanced in Theoretical and Applied Mathematics, 6, 13-18.

[9] Elkazi, T.M., Elkazi, S.M. and Hilal, E.M.A. (2012) Elkazi and Sumudu Transforms for Solving Some Differential Equations. Global Journal of Pure and Applied Mathematics, 2, 167-173.

[10] O'connor, J.J. and Robertson, E.F. (1996) Jacopo Francesco Riccati. http://www-history.mcs.st-andrews.ac.uk/Biographies/Riccati.html

[11] Schuch, D. (2014) Nonlinear Riccati Equations as a Unifying Link between Linear Quantum Mechanics and Other Fields of Physics. Journal of Physics. Conference Series, 504, Article ID: 012005. https://doi.org/10.1088/1742-6596/504/1/012005

[12] Haaheim, D.R. and Stein, F.M. (1969) Methods of Solution of the Riccati Differential Equation. Mathematics Magazine, 42, 233-240. https://doi.org/10.1080/0025570X.1969.11975969 